\title{
Subjects With COPD Walk With Less Consistent Organization of Movement Patterns of the Lower Extremity
}

\author{
Wai-Yan Liu, Kendra K Schmid, Kenneth Meijer, Martijn A Spruit, and Jennifer M Yentes
}

\begin{abstract}
BACKGROUND: The inherent stride-to-stride fluctuations during walking are altered in the aging population and could provide insight into gait impairments and falls in patients with COPD. Stride-to-stride fluctuations are quantified two ways: variability of the fluctuations (eg, standard deviation), and movement patterns within the fluctuations. Our objective was to investigate strideto-stride fluctuations by evaluating the variability and movement patterns of lower limb joints in subjects with COPD compared to subjects without COPD as control subjects. METHODS: In this cross-sectional study, 22 subjects with COPD (age $63 \pm 9$ y; FEV $54 \pm 19 \%$ predicted) and 22 control subjects (age $62 \pm 9 \mathrm{y} ; \mathrm{FEV}_{1} 95 \pm 18 \%$ predicted) walked for $3 \mathrm{~min}$ on a treadmill while their gait was recorded. The amount of variability (ie, standard deviation and coefficient of variation) and movement patterns (ie, predictability and consistency in organization) were quantified for the range of motion and joint angle of the hip, knee, and ankle, at 3 walking speeds (ie, self-selected, fast, and slow). General linear mixed models were used for analysis. RESULTS: Control subjects had more consistent organization of the hip and knee joint movement patterns compared to subjects with COPD $(P=.02$ and $P=.02$, respectively $)$. Further, control subjects adapted to speed changes by demonstrating more consistent organization of movement patterns with faster speeds, whereas subjects with COPD did not. At the fast walking speed, subjects with COPD demonstrated less consistent organization of knee and hip joint movement patterns as compared to control subjects without COPD $(P=.03$ and $P=.005$, respectively $)$. The amount of variability did not differ between groups. CONCLUSIONS: Although subjects with COPD did not demonstrate decreased amount of variability, their hip and knee joint movement patterns were less consistent in organization during walking. Reduced consistency in organization of movement patterns may be a contributing factor to falls and mobility problems experienced by patients with COPD. Key words: gait; lung disease; entropy; Lyapunov exponent; biomechanics; 3-dimensional motion analysis. [Respir Care 2020;65(2):158-168. (C) 2020 Daedalus Enterprises]
\end{abstract}

\section{Introduction}

COPD is characterized by progressive and persistent expiratory air-flow limitations associated with chronic in-

\footnotetext{
Ms Liu and Dr Spruit are affiliated with the Department of Research and Education, CIRO, Haelen, The Netherlands. Ms Liu and Dr Meijer are affiliated with Department of Nutrition and Movement Sciences, and Dr Spruit is affiliated with the Department of Respiratory Medicine, NUTRIM School of Nutrition and Translational Research in Metabolism, Maastricht University Medical Centre, Maastricht, The Netherlands. Dr Schmid is affiliated with the Department of Biostatistics, University of Nebraska Medical Center, Omaha, Nebraska. Dr Spruit is affiliated with REVAL Rehabilitation Research Center, BIOMED - Biomedical Research Institute, Faculty of Rehabilitation Sciences, Hasselt University, Diepenbeek, Belgium. Dr Yentes is affiliated with the Department of Biomechanics
}

flammation of the airway. ${ }^{1}$ Individuals with COPD are limited in their functional mobility and demonstrate lower limb muscle dysfunction. ${ }^{2,3}$ In addition, gait abnormalities have been observed in patients with COPD, including loss of function at the ankle joint, slower cadence, shorter step length, and narrower step width. ${ }^{4-8}$

Gait abnormalities can also be described by the inherent stride-to-stride fluctuations in gait parameters during walk-

\footnotetext{
and Center for Research in Human Movement Variability, University of Nebraska at Omaha, Omaha, Nebraska.

This work was supported by grants from the National Institutes of Health (P20 GM109090) and the European Respiratory Society (Fellowship STRTF 2016).
} 
ing. ${ }^{9}$ Traditional methods quantify the variability, or the amount, of stride-to-stride fluctuations (eg, the standard deviation or coefficient of variation). Complementary to traditional methods, other methods quantify movement patterns within the stride-to-stride fluctuations, such as how repeatable the fluctuations are within a walking bout. There are several methods available to quantify the movement patterns within stride-to-stride fluctuations. Sample entropy quantifies the predictability of the movement patterns by measuring the probability that patterns will not be followed by additional similar patterns. ${ }^{10}$ This method has been used to identify abnormal heart rate characteristics in patients with COPD. ${ }^{11}$ The local divergence exponent measures the consistency in the organization of movement patterns over time (referred to as local dynamic stability). ${ }^{12}$ Less consistent organization of movement patterns during walking has been reported in fall-prone elderly subjects. ${ }^{13}$ Quantifying movement patterns is important because flexibility from stride to stride is needed to execute adaptations to the changing environment while walking. ${ }^{14}$ Disease and aging have been associated with a loss of flexibility, reflected by movement patterns that are either too rigid or too irregular. ${ }^{15}$

There is little research on stride-to-stride fluctuations in patients with COPD. A few studies have reported reduced variability in step width and increased variability in step time and stride lengths in subjects with COPD. ${ }^{8,16}$ Similar gait changes have been related to increased fall risk in older adults. ${ }^{17,18}$ However, these spatiotemporal gait variables provide only a snapshot of gait. Joint movements may provide a more global view of the lower extremity movements. ${ }^{8}$ Previous studies have described altered lower limb joint movement patterns in older adults compared to young adults, and in subjects with peripheral arterial disease compared to healthy subjects. ${ }^{19,20}$ In addition, variability in spatiotemporal gait variables is not always discriminative between healthy older adults and older adults with a cautious gait. ${ }^{21}$ Therefore, quantifying joint movement patterns in patients with COPD could provide additional insight into gait alterations and might explain increased balance disturbances, fall risk, and mobility problems in this population. ${ }^{22,23}$

The aim of this study was to investigate stride-to-stride fluctuations by evaluating the variability and the move-

Supplementary material related to this paper is available at http:// www.rcjournal.com.

Correspondence: Jennifer M Yentes PhD, Department of Biomechanics and Center for Research in Human Movement Variability, University of Nebraska at Omaha, 6160 University Drive South, Omaha, Nebraska 68281-0860. E-mail: jyentes@gmail.com.

DOI: $10.4187 /$ respcare.06743

\section{QUICK LOOK}

\section{Current knowledge}

Stride-to-stride fluctuations are inherent during walking and can be quantified by the amount of fluctuations or the patterns within the fluctuations. The patterns within strideto-stride fluctuations during walking reflect the flexibility of the locomotor system to adapt during walking. Both the amount and patterns of stride-to-stride fluctuation are altered in the aging population and in patients with increased fall risk, and could provide insight into gait impairments and falls in patients with COPD.

\section{What this paper contributes to our knowledge}

The amount of stride-to-stride fluctuation did not differ between subjects with COPD and control subjects. However, subjects with COPD walked with less consistent organization of movement patterns of the lower extremities across walking speeds. These findings suggest the presence of neuromuscular deterioration in the locomotor system, reflecting less healthy movement patterns in subjects with COPD.

ment patterns in lower limb joints of subjects with COPD compared to control subjects without COPD while walking. We hypothesized that subjects with COPD would demonstrate a more restricted and a less consistent organization of gait patterns compared to control subjects, as reflected by decreased variability, increased predictability, and reduced consistency in organization in lower limb joint movement. To reveal adaptation needed to accommodate to alterations in demand, subjects were also asked to walk outside of their comfortable walking speed.

\section{Methods}

\section{Subjects}

In this cross-sectional study, subjects with COPD were recruited from the University of Nebraska Medical Center and the Omaha Veterans' Affairs Healthcare Center. COPD was previously diagnosed by a physician and confirmed with spirometry, using a $\mathrm{FEV}_{1} / \mathrm{FVC}$ of $<0.7 .{ }^{1} \mathrm{FEV}_{1} \%$ predicted was calculated using the reference data source of the NHANES III survey. ${ }^{24}$ Healthy control subjects were recruited through the community in Omaha and were considered healthy if they had an $\mathrm{FEV}_{1} / \mathrm{FVC}>0.7$. Individuals were excluded if they presented with a history of injury or disease that affected their mobility or another comorbidity that may affect the musculoskeletal, neurological, pulmonary, or cardiovascular systems. The insti- 
Table 1. Demographics of Subjects Used for Analysis

\begin{tabular}{|c|c|c|c|}
\hline Parameter & Control & COPD & $P$ \\
\hline Age, y & $62.1 \pm 8.6$ & $62.7 \pm 9.0$ & .83 \\
\hline Male/female, $n$ & $6 / 16$ & $13 / 9$ & $.033^{*}$ \\
\hline $\mathrm{FEV}_{1}, \mathrm{~L}$ & $2.4 \pm 0.6$ & $1.8 \pm 0.8$ & .01 \\
\hline FVC, L & $3.1 \pm 0.7$ & $3.2 \pm 1.1$ & .70 \\
\hline $\mathrm{FEV}_{1} / \mathrm{FVC}$ & $0.78 \pm 0.06$ & $0.55 \pm 0.12$ & $<.001$ \\
\hline $\mathrm{FEV}_{1} \%$ predicted & $94.5 \pm 17.7$ & $53.7 \pm 18.5$ & $<.001$ \\
\hline Height, cm & $165.0 \pm 9.4$ & $172.5 \pm 13.3$ & .038 \\
\hline Weight, $\mathrm{kg}$ & $73.6 \pm 15.6$ & $94.5 \pm 30.7$ & .007 \\
\hline Body mass index, $\mathrm{kg} / \mathrm{m}^{2}$ & $27.0 \pm 4.9$ & $31.6 \pm 8.7$ & .049 \\
\hline Slow, $\mathrm{m} / \mathrm{s}$ & $0.75 \pm 0.13$ & $0.68 \pm 0.21$ & .23 \\
\hline SSWS, m/s & $0.93 \pm 0.16$ & $0.85 \pm 0.26$ & .23 \\
\hline Fast, $\mathrm{m} / \mathrm{s}$ & $1.12 \pm 0.19$ & $1.02 \pm 0.31$ & .23 \\
\hline \multicolumn{4}{|c|}{$\begin{array}{l}\text { Data are presented as mean } \pm \text { SD or as } n \text { subjects. Control group: } n=22 \text { subjects; COPD } \\
\text { group: } n=22 \text { subjects. } \\
\text { * An association was found between gender and group (chi-square }(1)=4.539, P=.033 \text { ). } \\
\text { SSWS = self-selected walking speed }\end{array}$} \\
\hline
\end{tabular}

tutional review boards at both institutions approved the study, and the study was performed at the Department of Biomechanics and Center for Research in Human Movement Variability, University of Nebraska at Omaha. All subjects provided written informed consent. In total, 22 subjects with COPD and 22 control subjects without COPD were included (Table 1). This sample size was based upon a pilot study of stride-to-stride fluctuations of step length and step time in subjects with COPD prior to the current study.

\section{Data Collection}

All subjects wore a tight-fitting suit (ie, wrestling singlet). Retroreflective markers were placed on bony landmarks of the body, bilaterally, according to a modified Helen Hayes marker set. ${ }^{25}$ Prior to data collection, subjects were asked to walk on a treadmill to determine their selfselected walking speed (SSWS). The procedure to assess the SSWS included a walking trial in which, when a subject indicated that a speed was comfortable, the subject continued to walk at that speed for $1 \mathrm{~min}$, at which time the subject was asked again if the speed was too fast or too slow. If the subject indicated it was too fast, the treadmill was slowed or vice versa. This continued until a comfortable speed was found, which was similar to the subject's normal pace. This procedure may have taken up to $15 \mathrm{~min}$, in which subjects also became accustomed to treadmill walking itself. The SSWS corresponded to the subject's normal pace, such as when they would walk from the car into a building. Subjects were then given a minimum 5-min rest to prevent fatigue. Subjects completed 3.5-min trials at their SSWS, in which data were recorded during the last 3 minutes. Subjects completed 2 additional trials at

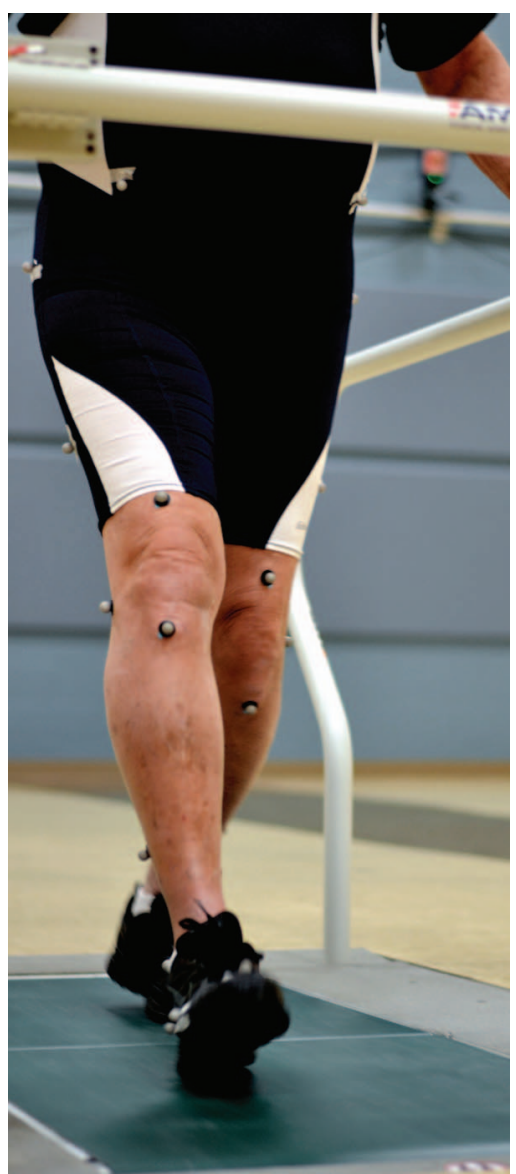

Fig. 1. Gait assessment setup. Infrared cameras are placed throughout the laboratory. Calibrated cameras triangulate the position of each marker based on the reflection of infrared light back to the camera lens off the retroflective marker. The positions of markers were used to calculate joint angles and range of motion of the joints.

speeds $-20 \%$ (slow) and $+20 \%$ (fast) of their SSWS. The order of the latter 2 walking speeds was randomized for all subjects. Subjects rested a minimum of $2 \mathrm{~min}$ to recover between trials.

Three-dimensional marker trajectories were collected with a high-speed motion capture system (Motion Analysis, Santa Rosa, California; $60 \mathrm{~Hz}$ ) while subjects walked on a treadmill (Fig. 1). Unfiltered 3-dimensional marker data were used to calculate sagittal joint angle time series for the ankle, knee, and hip (Visual 3D, C-Motion, Germantown, Maryland). The range of motion (RoM) was calculated for every right and left step from the joint angle time series using a custom Matlab program (Mathworks, Natick, Massachusetts) (Fig. 2). Generated time series for RoM data were cut to 162 steps. This was based upon the subject who had the lowest number of steps of all trials. To quantify the variability of fluctuations, we calculated standard deviations and coefficients of variation from the RoM time series. To quantify the movement patterns, RoM time 

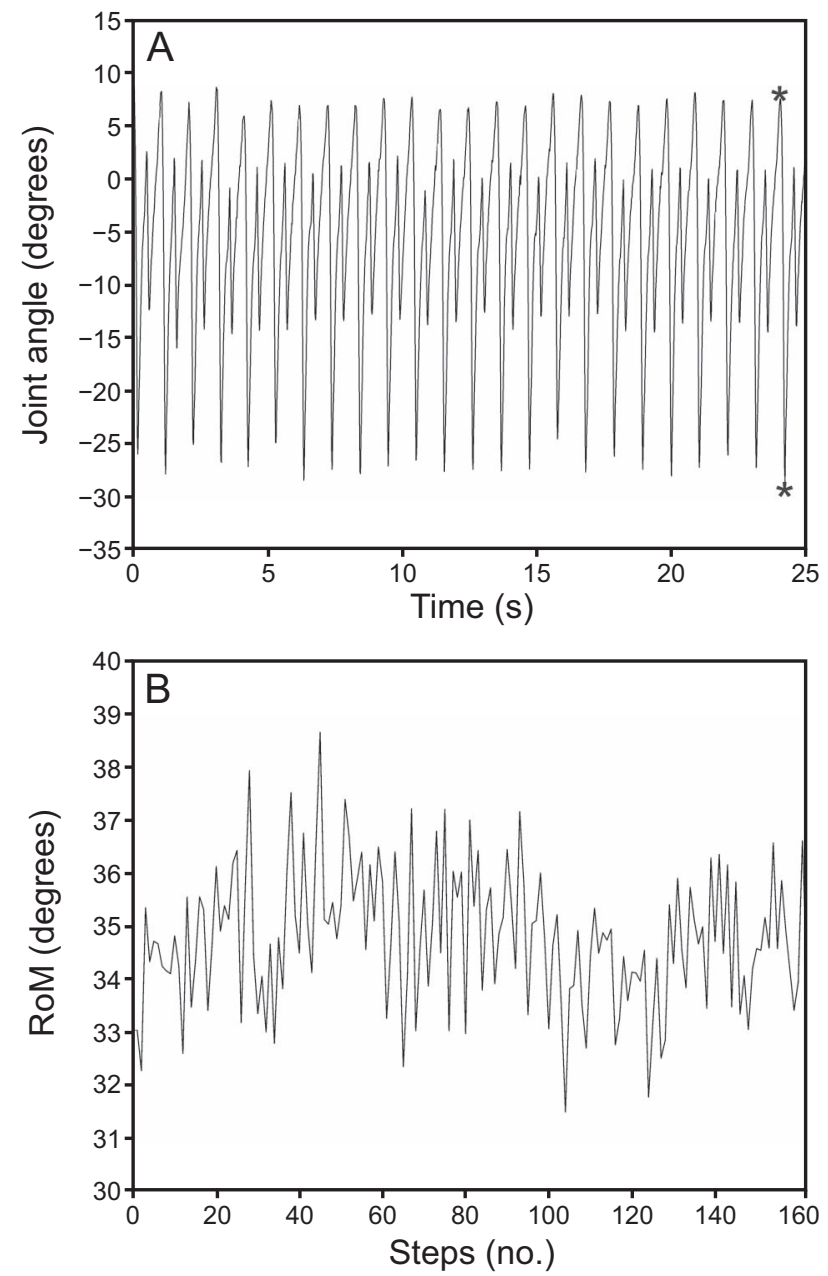

Fig. 2. A graphic representation of the joint angle and range of motion time series of the right ankle of a healthy subject. A: Represents a selection of the joint angle time series of the ankle (0-25 s). The asterisks reflect the maximum and minimum value of the joint angle in one stride, (e.g. the range of motion in one stride; RoM). B: Represents the RoM time series calculated over the entire joint angle time series.

series were subjected to predictability analysis. In addition, the right leg joint angles from the ankle, knee, and hip were subjected to consistency of organization analysis.

Sample entropy was used to assess the predictability of the RoM time series as described elsewhere. ${ }^{10}$ Briefly, sample entropy compares patterns within the time series to determine the probability that the same pattern will not appear again in the time series. A sample entropy value near zero reflected a near-perfect predictable time series of RoM values, whereas a sample entropy value extending toward infinity reflected a completely random time series of RoM values. A decrease in predictability has possible associations with a loss of flexible adaptation. Sample entropy was calculated using the $m$ parameter, which is the number of data points that are to be compared $(m=2)$, the $r$ parameter, which is the similarity criterion $(r=0.25)$, and the $n$ parameter, which is the length of the entire data set $(n=162)$, on the RoM time series for each joint (see the supplementary materials for justification of parameter selection at http://www.rcjournal.com). ${ }^{10}$

Lower limb joint angles were subjected to consistency of organization analysis (ie, local dynamic stability). ${ }^{26}$ To describe this analysis, imagine drawing a circle on a piece of paper over and over in a continuous fashion. Likely each iteration of the circle does not perfectly overlap the last circle. The further apart each iteration is, the less consistent the path is over time; if each iteration closely mimicked the iteration before, the path would be considered highly consistent. Similarly, this is true of the movement of the joint in space. Briefly, the joint angle time series was reconstructed into a multidimensional space, and the distances between these points were calculated as a function of time and averaged over all data point pairs to obtain the average logarithmic rate of divergence (Fig. 3). The slope of the divergence curve provided an estimate of the local divergence exponent, which quantifies the separation of the joint angle trajectories over time. A higher value of the local divergence exponent indicates a less consistent organization of movement pattern. ${ }^{27}$

Demographics were compared using a $t$ test or a chi-square test. A linear mixed model was used to assess differences in mean, standard deviation and coefficient of variation, and predictability of RoM time series between groups (COPD vs controls) and over the 3 walking speed conditions (slow, SSWS, and fast). A linear mixed model was used to determine group and speed differences of the consistency of organization in each joint angle (ankle, knee, and hip). This method enables the determination of differences within and between groups while accounting for correlation due to repeated measurements and adjusting for potentially confounding variables. All interactions between group and speed were investigated. Adjustments for multiple comparisons were made using the simulation technique. In all models, the actual speed at each tested pace (slow, SSWS and fast) was used as a covariate so comparisons could be made between speed groups independent of the starting speed. Mean differences between groups or speeds, 95\% CIs, and effect sizes (Cohen's $d$ ) were calculated. ${ }^{28}$ All statistical analyses were conducted in SAS (SAS Institute, Cary, North Carolina). A significance level of alpha $=0.05$ was set a priori.

\section{Results}

\section{Variability of Fluctuations}

A significant interaction between group and speed for mean ankle RoM and mean hip RoM was found $(P=.02$ and $P=.035$, respectively) (for additional information, see Fig. 4, Fig. 5, and the supplementary materials at http:// 

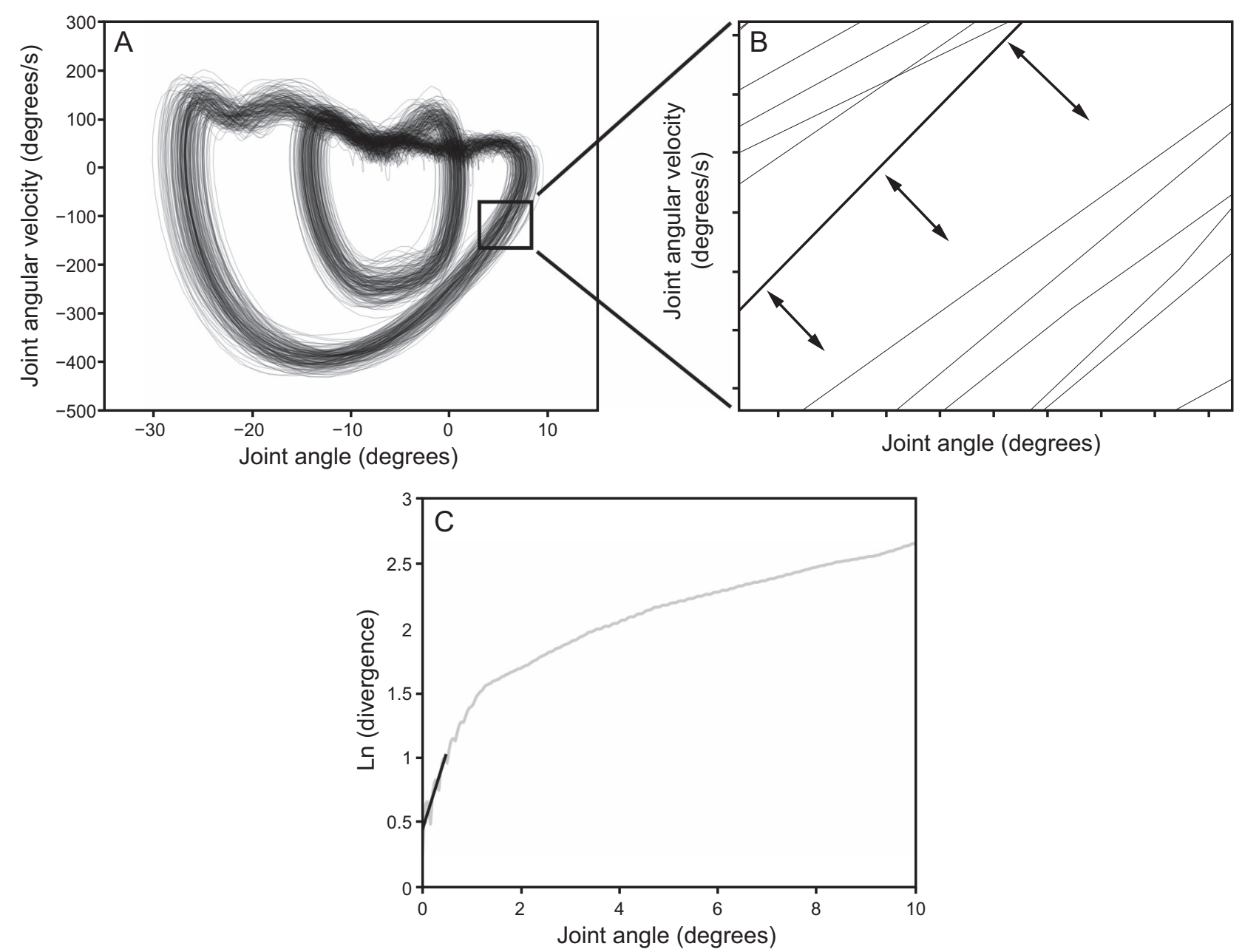

Fig. 3. A graphic representation of the ankle joint angle time series of a healthy subject in two dimensions and the calculation of the local divergence exponent. A: The phase portrait of a representative ankle joint angle (angle vs. angular velocity). B: A close-up view of one portion of the phase portrait where the divergence of neighboring trajectories is outlined. Distances between points were calculated as a function of time. C: The local divergence exponent is calculated as the slope of the strides vs. the natural log of the divergence plot at $0-0.5$ strides (grey line). To reconstruct the angles in a multi-dimensional space, time delay of 10 points ${ }^{47}$ and embedding dimension (ankle and knee: 7; hip: 6 dimensions) ${ }^{48}$ were calculated and used. Each joint angle time series was normalized to 100 data points per stride.

www.rcjournal.com). Subjects with COPD and control subjects appeared to have a similar ankle and hip RoM at slow speed, although only control subjects seemed to increase their RoM with faster speeds.

Knee RoM standard deviation demonstrated a main effect of speed for both groups combined $(P=.02)$ (Fig. 6). Knee RoM was more variable in the fast condition compared to SSWS $(P=.01)$ and slow condition $(P=.049)$ (Table 2$)$.

Similar to standard deviation, knee RoM coefficient of variation demonstrated a significant main effect of speed $(P=.01)$. Walking at the fast speed was more variable in knee RoM compared to SSWS $(P=.01, d=0.64)$ and slow speed $(P=.02)$. A main effect of speed on hip RoM coefficient of variation was found $(P=.036)$. Walking at the fast speed was more variable in hip RoM compared to SSWS $(P=.046)$ and slow speed $(P=.033)$.

\section{Movement Patterns Within the Fluctuations}

No main effect of group was found for predictability across any of the joints. A main effect of speed for hip RoM predictability was found $(P=.02)$. During the fast speed, hip RoM was more predictable compared to SSWS $(P=.049)$ and slow speed $(P=.01)$. No interactions were found.

A significant interaction between group and speed was found in the consistency in the organization of the knee $(P=.02)$ and hip $(P=.02)$ joint angles. Control subjects exhibited increased consistency in organization (eg, lower local divergence exponent values) in the hip and knee angle movement patterns as speed increased, while subjects with COPD did not demonstrate as much improvement. In the fast condition, subjects with COPD showed significantly reduced 

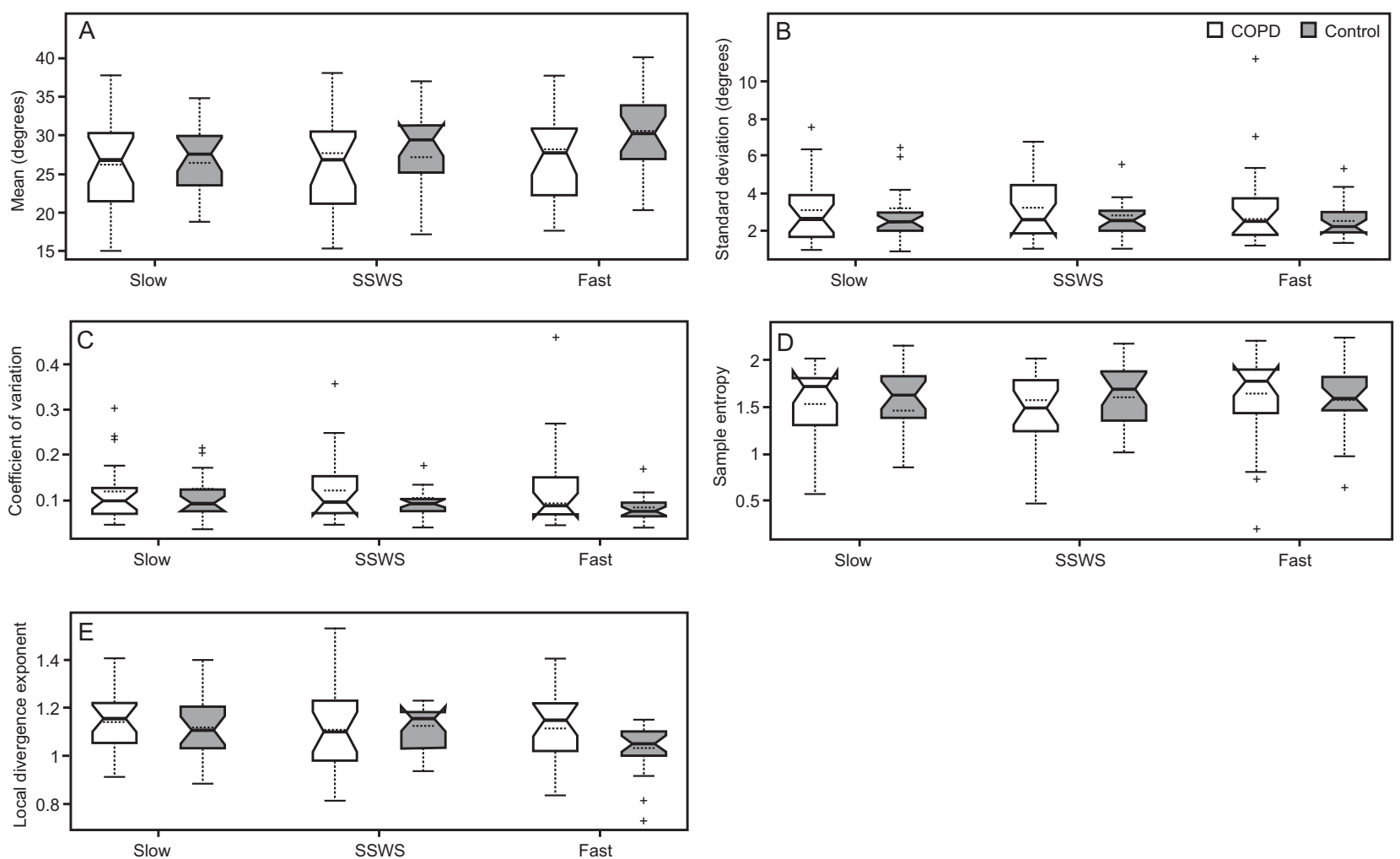

Fig. 4. Box plots of the gait parameters of the ankle joint between subjects with COPD and control subjects. A: Mean ankle range of motion; $\mathrm{B}$ : standard deviation of the ankle range of motion; C: coefficient of variation of the ankle range of motion; D: predictability of the ankle range of motion; and E: consistency in the organization of the ankle joint angle. Centerlines show the medians, dotted centerlines show the means; box limits indicate the 25th and 75th percentiles; whiskers extend to the most extreme data points not considered outliers; outliers are plotted using the '+' symbol.

consistency in organization of the knee joint angle as compared to the control subjects $(P=.03)$ and for the hip joint angle $(P=.005)$. Within the COPD group, hip joint angles demonstrated reduced consistency in organization (eg, greater local divergence exponent values) in the fast speed as compared to the SSWS and slow speed $(P=.045$ and $P=.045$, respectively).

\section{Discussion}

This study aimed to investigate stride-to-stride fluctuations by evaluating the variability and movement patterns in lower limb joints between the walking gait of subjects with COPD and control subjects who did not have COPD. We hypothesized that subjects with COPD would demonstrate more restricted and less consistent organization of movement patterns, as reflected by a reduced variability of fluctuations, increased predictability, and reduced consistency in the organization of joint movement patterns within the stride-to-stride fluctuations. In addition, it was hypothesized that this would become more apparent at walking speeds that differed from the SSWS.
Data partially supported our hypothesis. In agreement, subjects with COPD demonstrated less consistent organization of the knee and hip joint angles compared to control subjects. However, control subjects adapted to speed changes by demonstrating more consistent organization of movement patterns at faster speeds than subjects with COPD did. During fast walking, subjects with COPD showed less consistency in the organization of both knee and hip joint angles compared to controls, suggesting that gait instability impairments in the locomotor system become apparent when accommodating to increase in task demands. In contrast to our hypothesis, no differences between groups were found for the variability of fluctuations or for predictability.

Healthy joint angle movement patterns represent a coordinated locomotor system able to make flexible adaptations to demands placed on the body during walking. ${ }^{9}$ Subjects with COPD might have been unable to select the required response when forced to accommodate to alterations in task demands (eg, a change from SSWS). ${ }^{20} \mathrm{Re}-$ duced consistency in the organization of joint movement patterns suggests the presence of neuromuscular deterio- 

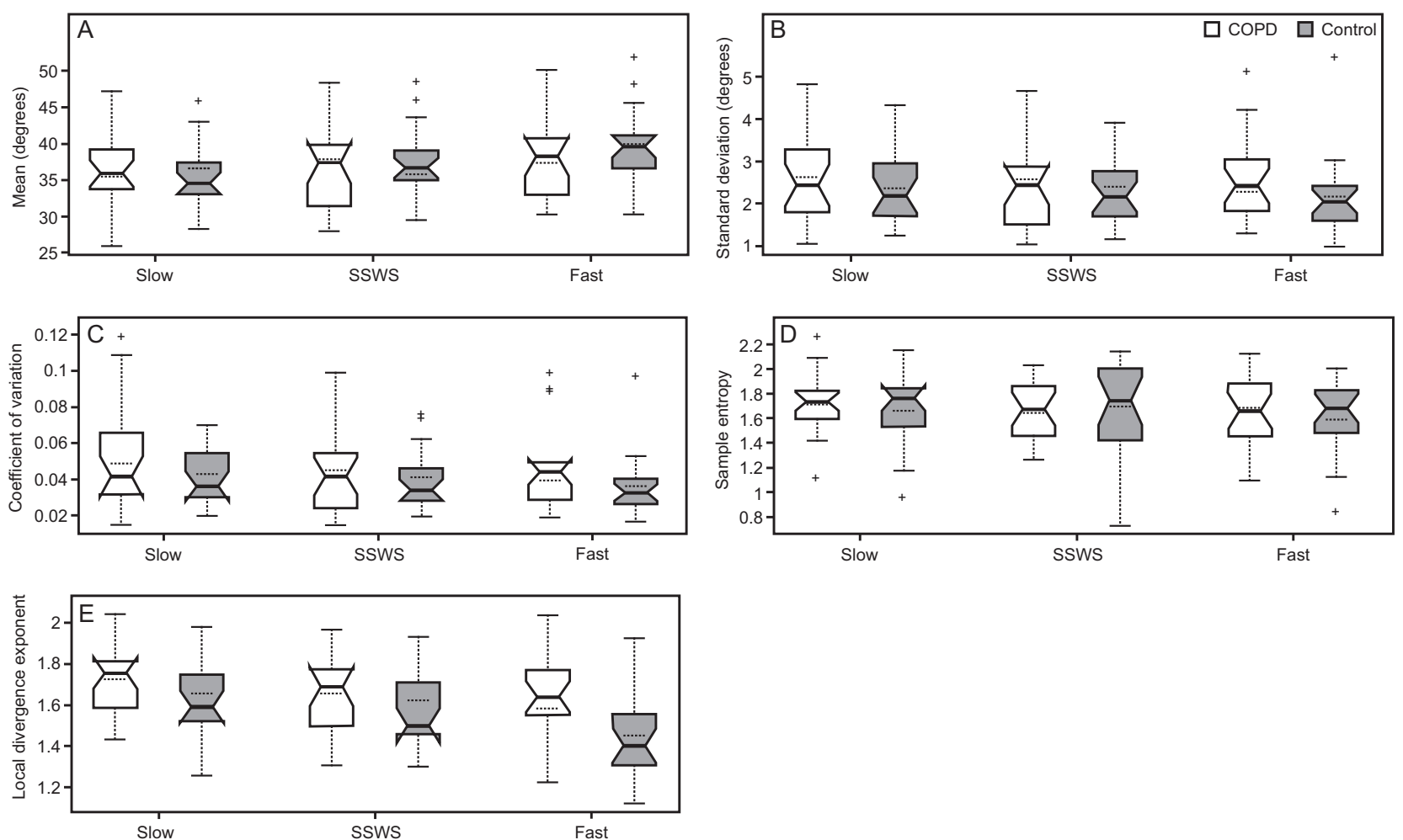

Fig. 5. Box plots of the gait parameters of the hip joint between subjects with COPD (white) and control subjects (gray). A: Mean hip range of motion; B: standard deviation of the hip range of motion; C: coefficient of variation of the hip range of motion; D: predictability of the hip range of motion; E: consistency in the organization of the hip joint angle. Centerlines show the medians, dotted centerlines show the means; box limits indicate the 25th and 75th percentiles; whiskers extend to the most extreme data points not considered outliers; outliers are plotted using the '+' symbol.

ration in the locomotor system, reflecting less healthy movement patterns. ${ }^{20}$ Reduced consistency in the organization of movement patterns during walking are present in patients with peripheral arterial disease ${ }^{20}$ and fall-prone elderly patients. ${ }^{13}$

In subjects with COPD, reduced consistency in the organization of the joint movement patterns could be related to extrapulmonary manifestations, such as skeletal muscle dysfunction present in COPD. ${ }^{3} \mathrm{~A}$ loss of muscle mass and strength in the lower limb muscles has been found in individuals with COPD, including the quadriceps, the tibialis anterior, and the gastrocnemius. ${ }^{29,30}$ Alterations in these skeletal muscles are especially important because these muscles are essential for walking and adjusting to task and environmental demands. Therefore, reduced strength in the lower limb muscles could be associated with gait alterations and the accompanying stride-to-stride fluctuations. ${ }^{5-8,16}$ In addition, in patients with COPD, the lack of ability to adjust their movement patterns with changes in walking speed, may be associated with an increased susceptibility for balance disturbances and falls, which has been reported in this patient population. ${ }^{22,23}$

Subjects in this study did not demonstrate a more restricted gait, as reflected by a reduced variability of fluc- tuations, compared to control subjects. A possible explanation could be that variability is a measure of magnitude and may not be sensitive enough to changes in fluctuations between groups, unless these changes are drastic or have a lot of outliers. ${ }^{31}$ Our results showed that subjects with COPD did not differ in predictability of movement patterns from control subjects, whereas the consistency in the organization of movement patterns was different between the groups. A possible explanation could be that predictability and consistency measures quantify different aspects of movement patterns; both are related to the flexibility of the locomotor system as found in healthy subjects. In addition, conflicting results have been reported on sample entropy in COPD. Yentes et $\mathrm{a}^{8}$ reported that predictability in step time and step width was not sensitive enough to differentiate between subjects with COPD and subjects without COPD. However, differences between subjects with COPD and healthy subjects were reported in heart rate and air-flow patterns. ${ }^{11,32}$ Less consistency in the organization of movement patterns was found in various patient populations as compared to healthy older adults, ${ }^{12,13,20}$ although this measure has not been applied in subjects with COPD. Because existing knowledge of variability of and patterns within stride-to-stride fluctua- 

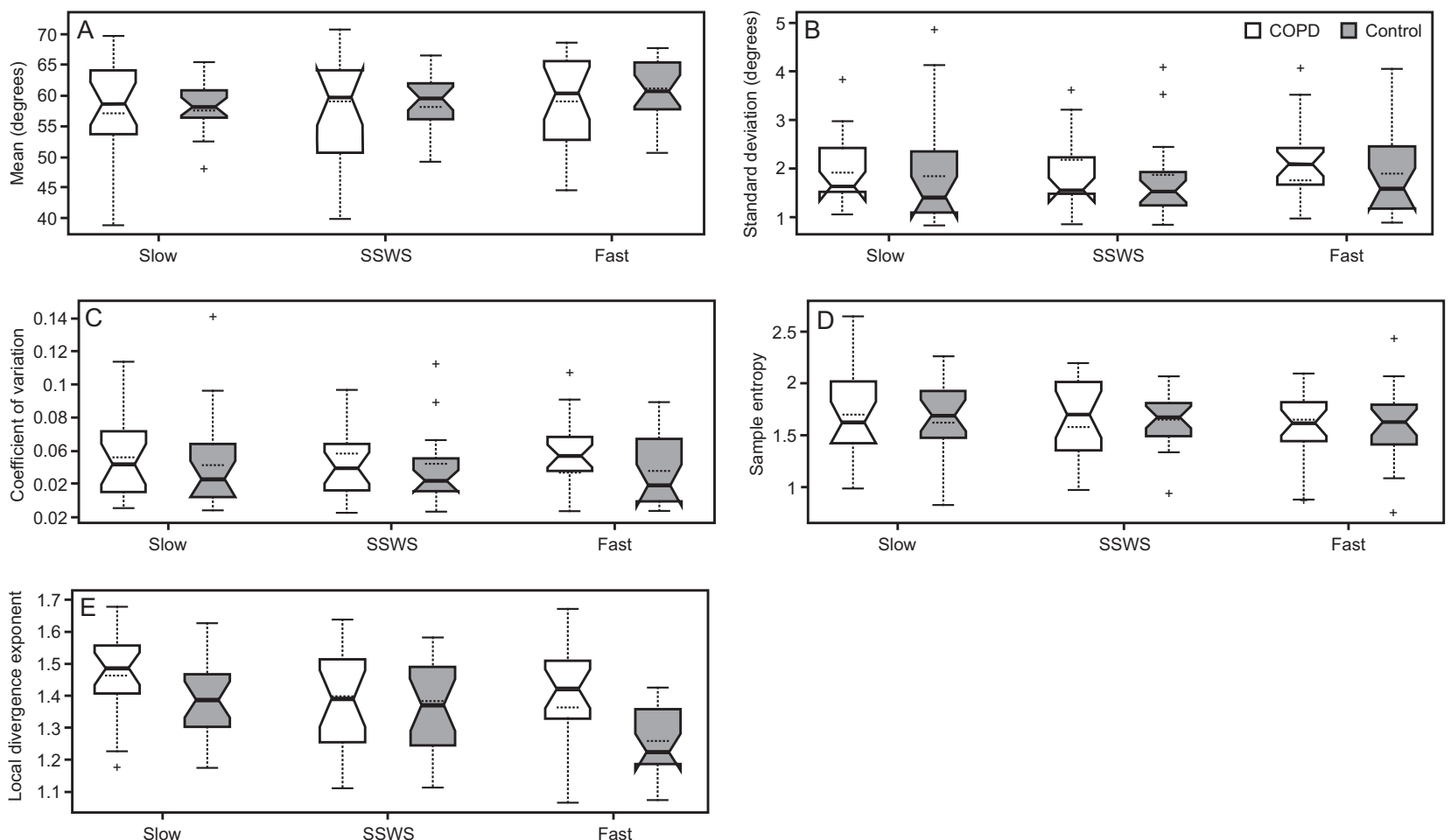

Fig. 6. Box plots of the gait parameters of the knee joint between subjects with COPD (white) and control subjects (gray). A: Mean knee range of motion; B: standard deviation of the knee range of motion; C: coefficient of variation of the knee range of motion; D: predictability of the knee range of motion; E: consistency in the organization of the knee joint angle. Centerlines show the medians, dotted centerlines show the means; box limits indicate the 25th and 75th percentiles; whiskers extend to the most extreme data points not considered outliers; outliers are plotted using the '+' symbol.

Table 2. Significant Adjusted Post-Hoc Analyses

\begin{tabular}{|c|c|c|c|c|}
\hline Parameter & Comparison & Mean Difference $(95 \% \mathrm{CI})$ & Effect Size & $P$ \\
\hline \multirow[t]{2}{*}{ Standard deviation of knee RoM } & Fast vs SSWS & $0.40(0.08-0.72)$ & 0.63 & .01 \\
\hline & Fast vs Slow & $0.46(0.00-0.92)$ & 0.51 & .049 \\
\hline \multirow[t]{2}{*}{ Coefficient of variation of knee RoM } & Fast vs SSWS & $0.01(0.00-0.02)$ & 0.64 & .01 \\
\hline & Fast vs Slow & $0.01(0.00-0.03)$ & 0.57 & .02 \\
\hline \multirow[t]{2}{*}{ Coefficient of variation of hip RoM } & Fast vs SSWS & $0.01(0.00-0.01)$ & 0.51 & .046 \\
\hline & Fast vs Slow & $0.01(0.00-0.02)$ & 0.54 & .033 \\
\hline \multirow[t]{2}{*}{ Predictability of hip RoM } & Fast vs SSWS & $-0.11(-0.23-0.00)$ & 0.50 & .049 \\
\hline & Fast vs Slow & $-0.20(-0.37$ to -0.04$)$ & 0.62 & .01 \\
\hline Consistency in organization of knee joint angles & COPD vs control subjects at Fast & $0.11(0.01-0.21)$ & 0.91 & .03 \\
\hline Consistency in organization of hip joint angles & COPD vs control subjects at Fast & $0.15(0.03-0.28)$ & 1.07 & .005 \\
\hline $\begin{array}{l}\text { Consistency in organization of hip joint angles } \\
\text { in COPD }\end{array}$ & Fast vs Slow & $0.10(0.00-0.19)$ & 0.85 & .045 \\
\hline $\begin{array}{l}\text { Consistency in organization of hip joint angles } \\
\text { in COPD }\end{array}$ & Fast vs Slow & $0.11(0.00-0.23)$ & 0.85 & .045 \\
\hline $\begin{array}{l}\text { RoM }=\text { range of motion } \\
\text { SSWS }=\text { self-selected walking speed } \\
\text { Fast }=20 \% \text { faster than SSWS } \\
\text { Slow }=20 \% \text { slower than SSWS }\end{array}$ & & & & \\
\hline
\end{tabular}

tions in patients with COPD is limited, these measures should be used to complement each other to gain further insight into gait alterations in COPD.
The patterns of the joint movement pattern within the strides differed between the groups as measured by the consistency in the organization of movement patterns. There 
are 2 likely explanations for these differences: biomechanical limits of the joint movement, and the nature of the data that was used in each method. First, the anatomy of the human body limits the RoM. Consequently, the RoM is less variable due to the anatomical limits of the minimum and maximum RoM for a given joint. However from minimum to maximum, the joint movement can be variable and fluctuate, and this may provide information about the joint movement pattern that the RoM does not take into account. Second, only continuous joint angle data demonstrated differences between groups as compared to the discrete RoM data. Discrete time series (ie, time series of distinct events within the gait cycle) are more appropriate for the calculation of sample entropy. Therefore, we used RoM time series for this analysis. ${ }^{14}$ The consistency in organization analysis was calculated using the continuous data of joint angles, which is more traditional for this measure. Consequently, consistency measures in joint angle time series may be more appropriate to differentiate gait alterations in COPD from controls subjects. Further research is warranted to identify appropriate measures to assess movement patterns in COPD and the clinical importance of these measures.

Our study supports the effect of walking speed on both the variability and movement patterns of stride-to-stride fluctuations. ${ }^{33-39}$ Variability of fluctuations in the knee and hip joint RoM was greatest at the fast walking speeds in both groups; that is, variability was greater in the fast speed compared to both the SWSS and slow speeds. However, no consistent relationship was found for the variability of stride-to-stride fluctuations and walking speed; whereas, other studies have reported a negative linear relationship ${ }^{33,35}$ or a U-shaped relationship. ${ }^{34,36}$ One study by Kang and Dingwell ${ }^{40}$ reported a trend of higher (though not statistically significant) mean standard deviations in knee and hip flexion-extension, as well as ankle plantarflexion-dorsiflexion, in older adults with increasing walking speed (from preferred walking speed to $+20 \%$ of preferred walking speed). This is similar to the changes in RoM observed in our study. A possible explanation could be that faster walking speeds posed increased difficulty for the subject, which resulted in increased variability at fast walking speed. Similar to variability, walking speed alters movement patterns within stride-to-stride fluctuations. ${ }^{34,35,37-39}$ Our findings indicated an increased predictability of the hip RoM during fast walking, while Costa et $\mathrm{al}^{37}$ reported their highest predictability values during walking at a normal speed. A possible explanation for these differences may lie in the difference between overground walking, as used by Costa et al, ${ }^{37}$ and treadmill walking, as used in our study. In addition, previous studies have reported reduced consistency in the organization of gait movement patterns with increasing walking speeds..$^{38,39}$ This contrasts with our findings, as no clear trend was found in the consistency in the organization of the joint angles over the walking speeds. However, differences in walking speed might have affected these results because our subjects walked at their SSWS; whereas, one other study based the walking speed on fast walking determined during the 6-min walk test, ${ }^{39}$ while another study scaled the walking speed to leg length and pendulum dynamics. ${ }^{38}$

This study has some limitations. The use of $\mathrm{FEV}_{1} / \mathrm{FVC}<$ 0.70 as confirmatory of air-flow obstruction may misclassify some individuals in this study. However, all subjects in the COPD group also had a physician diagnosis of COPD. In addition, a potential effect of the male to female ratio, height, weight, and body mass index on the study results should be mentioned, as differences were found between COPD and control subjects. In the current study, we did not record whether subjects with COPD used a bronchodilator prior to their visit to our research facility. In addition, subjects with COPD have a reduced walking capacity due to the pathophysiology of the disease. The duration of treadmill walking is therefore limited, resulting in shorter data lengths per trial in which the biological complexity of the movement pattern could not be captured. However, sample entropy and local divergence exponents are quite robust against different data lengths and tend to respond better to short data lengths. ${ }^{10,41}$ Another limitation is that the speed perturbations of $20 \%$ of the subject's SSWS may not have been challenging enough. Therefore, measures to assess the amount of variability and patterns within the stride-to-stride fluctuations to identify gait impairments may not have surfaced in the subjects with COPD. In addition, treadmill walking differs from overground walking, and the treadmill might have imposed a constraint on the subjects by limiting the fluctuations in walking that are normally present in overground walking. ${ }^{42}$ Little difference in joint angles have been reported between treadmill and overground walking, ${ }^{43}$ although it is possible that the stride-to-stride fluctuations of the data were affected by the use of the treadmill, as differences in stride-to-stride fluctuations in spatiotemporal gait parameters have been reported between overground and treadmill walking. ${ }^{16,44-46}$ Another limitation is the potentially heterogeneous sample of subjects with COPD in this study. We included a sample size of 44 (22 subjects per group). Therefore, further studies involving a larger population are suggested to determine additional parameters for gait impairments in subjects with COPD. Contractile fatigue occurs during exercise in some patients with COPD and may be a limiting factor in exercise tolerance. ${ }^{2}$ Subjects who experience exercise limitations by fatigue may have presented more gait impairments compared to subjects who are mainly limited by dyspnea. However, dyspnea and fatigue were not assessed in this study. Future studies are therefore recommended to investigate the association between the heterogeneity in COPD and gait 
impairments. Furthermore, the observations could not be related to COPD alone because body composition changes and differences in muscle mass of lower extremities may have affected the stride-to-stride fluctuations in subjects with COPD and control subjects, and these factors were not assessed in this study. Research on the contribution of body composition and muscle strength is therefore recommended for future studies.

\section{Conclusions}

Although subjects with COPD did not demonstrate decreased variability, subjects with COPD show reduced consistency in the organization of hip and knee joint angle patterns across the walking speeds. Reduced consistency in the organization of the hip and knee joint movements might be a contributing factor to falls and mobility problems experienced by subjects with COPD.

\section{REFERENCES}

1. Global Initiative for Chronic Obstructive Lung Disease. Global Strategy for the Diagnosis, Management, and Prevention of Chronic Obstructive Pulmonary Disease: 2018 Report. Available at: https:// www.goldcopd.org. Accessed February 2, 2019.

2. Saey D, Debigare R, LeBlanc P, Mador MJ, Cote CH, Jobin J, Maltais F. Contractile leg fatigue after cycle exercise: a factor limiting exercise in patients with chronic obstructive pulmonary disease. Am J Respir Crit Care Med 2003;168(4):425-430.

3. Mathur S, Brooks D, Carvalho CR. Structural alterations of skeletal muscle in copd. Front Physiol 2014;5:104.

4. Yentes JM, Schmid KK, Blanke D, Romberger DJ, Rennard SI, Stergiou N. Gait mechanics in patients with chronic obstructive pulmonary disease. Respir Res 2015;16:31.

5. Annegarn J, Spruit MA, Savelberg HH, Willems PJ, van de Bool C, Schols AM, et al. Differences in walking pattern during 6-min walk test between patients with COPD and healthy subjects. PLoS One 2012;7(5):e37329.

6. Lahousse L, Verlinden VJ, van der Geest JN, Joos GF, Hofman A, Stricker BH, et al. Gait patterns in COPD: the Rotterdam Study. Eur Respir J 2015;46(1):88-95.

7. Nantsupawat N, Lane P, Siangpraipunt O, Gadwala S, Nugent K. Gait characteristics in patients with chronic obstructive pulmonary disease. J Prim Care Community Health 2015;6(4):222-226.

8. Yentes JM, Rennard SI, Schmid KK, Blanke D, Stergiou N. Patients with chronic obstructive pulmonary disease walk with altered step time and step width variability as compared with healthy control subjects. Ann Am Thorac Soc 2017;14(6):858-866.

9. Stergiou N, Buzzi UH, Kurz MJ, Heidel J. Nonlinear tools in human movement. Champaign, IL: Human Kinetics Publishers, Inc.; 2004: 63-90.

10. Yentes JM, Hunt N, Schmid KK, Kaipust JP, McGrath D, Stergiou $\mathrm{N}$. The appropriate use of approximate entropy and sample entropy with short data sets. Ann Biomed Eng 2013;41(2):349-365.

11. Goulart CDL, Simon JC, Schneiders PDB, San Martin EA, Cabiddu R, Borghi-Silva A, et al. Respiratory muscle strength effect on linear and nonlinear heart rate variability parameters in COPD patients. Int J Chron Obstruct Pulmon Dis 2016;11:1671-1677.

12. Bruijn SM, Meijer OG, Beek PJ, van Dieen JH. Assessing the stability of human locomotion: a review of current measures. J R Soc Interface 2013;10(83):20120999.
13. Lockhart TE, Liu J. Differentiating fall-prone and healthy adults using local dynamic stability. Ergonomics 2008;51(12):1860-1872.

14. Stergiou N, Harbourne R, Cavanaugh J. Optimal movement variability: a new theoretical perspective for neurologic physical therapy. J Neurol Phys Ther 2006;30(3):120-129.

15. Vaillancourt DE, Newell KM. Changing complexity in human behavior and physiology through aging and disease. Neurobiol Aging 2002;23(1):1-11.

16. Liu WY, Spruit MA, Delbressine JM, Willems PJ, Franssen FME, Wouters EFM, Meijer K. Spatiotemporal gait characteristics in patients with COPD during the Gait Real-time Analysis Interactive Lab-based 6-minute walk test. PLoS One 2017;12(12):e0190099.

17. Brach JS, Berlin JE, VanSwearingen JM, Newman AB, Studenski SA. Too much or too little step width variability is associated with a fall history in older persons who walk at or near normal gait speed. J Neuroeng Rehabil 2005;2:21.

18. Callisaya ML, Blizzard L, Schmidt MD, Martin KL, McGinley JL, Sanders LM, Srikanth VK. Gait, gait variability and the risk of multiple incident falls in older people: a population-based study. Age Ageing 2011;40(4):481-487.

19. Buzzi UH, Stergiou N, Kurz MJ, Hageman PA, Heidel J. Nonlinear dynamics indicates aging affects variability during gait. Clin Biomech (Bristol, Avon) 2003;18(5):435-443.

20. Myers SA, Johanning JM, Stergiou N, Celis RI, Robinson L, Pipinos II. Gait variability is altered in patients with peripheral arterial disease. J Vasc Surg 2009;49(4):924-931e921.

21. Herman T, Giladi N, Gurevich T, Hausdorff JM. Gait instability and fractal dynamics of older adults with a "cautious" gait: why do certain older adults walk fearfully? Gait Posture 2005;21(2):178185 .

22. Crisan AF, Oancea C, Timar B, Fira-Mladinescu O, Tudorache V. Balance impairment in patients with COPD. PLoS One 2015;10(3): e0120573.

23. Roig M, Eng JJ, MacIntyre DL, Road JD, FitzGerald JM, Burns J, Reid WD. Falls in people with chronic obstructive pulmonary disease: an observational cohort study. Respir Med 2011;105(3):461469.

24. Hankinson JL, Odencrantz JR, Fedan KB. Spirometric reference values from a sample of the general U.S. population. Am J Respir Crit Care Med 1999;159(1):179-187.

25. Houck JR, Duncan A, De Haven KE. Knee and hip angle and moment adaptations during cutting tasks in subjects with anterior cruciate ligament deficiency classified as noncopers. J Orthop Sports Phys Ther 2005;35(8):531-540.

26. Bruijn SM, Bregman DJ, Meijer OG, Beek PJ, van Dieen JH. Maximum Lyapunov exponents as predictors of global gait stability: a modelling approach. Med Eng Phys 2012;34(4):428-436.

27. Stergiou N. Nonlinear analysis for human movement variability. Boca Raton: CRC Press; 2016:277-279.

28. Cohen J. Statistical power analysis for the behavioral sciences. New York: Routledge; 1988.

29. Donaldson AV, Maddocks M, Martolini D, Polkey MI, Man WD. Muscle function in COPD: a complex interplay. Int J Chron Obstruct Pulmon Dis 2012;7:523-535.

30. Gagnon P, Maltais F, Bouyer L, Ribeiro F, Coats V, Brouillard C, et al. Distal leg muscle function in patients with COPD. COPD 2013; 10(2):235-242.

31. Stergiou N, Decker LM. Human movement variability, nonlinear dynamics, and pathology: is there a connection? Hum Mov Sci 2011; 30(5):869-888.

32. Dames KK, Lopes AJ, de Melo PL. Airflow pattern complexity during resting breathing in patients with COPD: effect of airway obstruction. Respir Physiol Neurobiol 2014;192:39-47. 


\section{Stride-to-Stride Fluctuations in Subjects With COPD}

33. Chien JH, Yentes J, Stergiou N, Siu KC. The effect of walking speed on gait variability in healthy young, middle-aged and elderly individuals. J Phys Act Nutr Rehabil 2015;2015.

34. Dingwell JB, Marin LC. Kinematic variability and local dynamic stability of upper body motions when walking at different speeds. J Biomech 2006;39(3):444-452.

35. Jordan K, Challis JH, Newell KM. Walking speed influences on gait cycle variability. Gait Posture 2007;26(1):128-134.

36. Sekiya N, Nagasaki H, Ito H, Furuna T. Optimal walking in terms of variability in step length. J Orthop Sports Phys Ther 1997;26(5):266272.

37. Costa M, Peng CK, Goldberger AL, Hausdorff JM. Multiscale entropy analysis of human gait dynamics. Physica A 330(1-2):53-60, 2003.

38. England SA, Granata KP. The influence of gait speed on local dynamic stability of walking. Gait Posture 2007;25(2):172-178.

39. Manor B, Wolenski P, Li L. Faster walking speeds increase local instability among people with peripheral neuropathy. J Biomech 2008; 41(13):2787-2792.

40. Kang HG, Dingwell JB. Separating the effects of age and walking speed on gait variability. Gait Posture 2008;27(4):572-577.

41. Dingwell JB, Cusumano JP. Nonlinear time series analysis of normal and pathological human walking. Chaos 2000;10(4):848863.
42. Parvataneni K, Ploeg L, Olney SJ, Brouwer B. Kinematic, kinetic and metabolic parameters of treadmill versus overground walking in healthy older adults. Clin Biomech (Bristol, Avon) 2009;24(1): 95-100.

43. Riley PO, Paolini G, Della Croce U, Paylo KW, Kerrigan DC. A kinematic and kinetic comparison of overground and treadmill walking in healthy subjects. Gait Posture 2007;26(1):17-24.

44. Dingwell JB, Cusumano JP, Cavanagh PR, Sternad D. Local dynamic stability versus kinematic variability of continuous overground and treadmill walking. J Biomech Eng 2001;123(1):27-32.

45. Terrier P, Deriaz O. Kinematic variability, fractal dynamics and local dynamic stability of treadmill walking. J Neuroeng Rehabil 2011;8:12.

46. Hollman JH, Watkins MK, Imhoff AC, Braun CE, Akervik KA, Ness DK. A comparison of variability in spatiotemporal gait parameters between treadmill and overground walking conditions. Gait Posture 2016;43:204-209.

47. Thomas RD, Moses NC, Semple EA, Strang AJ. An efficient algorithm for the computation of average mutual information: validation and implementation in Matlab. J Math Psychol 2014; 61:45-59.

48. Kennel MB, Brown R, Abarbanel HD. Determining embedding dimension for phase-space reconstruction using a geometrical construction. Phys Rev A 1992;45(6):3403-3411.

This article is approved for Continuing Respiratory Care Education credit. For information and to obtain your CRCE

(free to AARC members) visit

www.rcjournal.com 